\title{
The relationship between academic self-efficacy and academic burnout in medical students
}

\author{
Su Hyun Lee and Woo Taek Jeon
}

Department of Medical Education, Yonsei University College of Medicine, Seoul, Korea

\section{의과대학생의 학업적 자기효능감과 학업 소진의 관계}

연세대학교 의과대학 의학교육학과

\section{이수현, 전우택}

Purpose: The purpose of this study was to examine the correlation between academic burnout and academic self-efficacy in medical students.

Methods: The study group comprised 446 students in years 1 to 4 of medical school. They were asked to rate their academic burnout and academic self-efficacy on a scale. The data were analyzed by multivariate analysis of variance and regression analysis. Results: Academic self-efficacy was correlated negatively with academic burnout explaining 37\% of academic burnout.

Conclusion: Academic self-efficacy (especially self-confidence) had the greatest effect on academic burnout. The implications of these results are discussed in terms of an evaluation and support system for students.

Key Words: Academic burnout, Academic self-efficacy, Self-confidence

\section{서론}

의과대학에 입학한 학생들을 소위 “공부의 신"이라고 부른 다. 그러나 이 학생들이 본과에 진입하면 학업으로 인한 심각 한 스트레스와 심리적 고통을 경험하곤 한다. 그 이유는 과중 한 학업량, 여가시간 부족, 좋은 성적 유지에 대한 심리적 압 박감, 환자와 일을 하면서 동시에 의학적 처치를 학습해야 하 는 복잡하고 특별한 학습 환경으로 다중적인 스트레스 요인 에 직면하기 때문이다[1,2].
스트레스를 설명하는 Conservation of Resource (COR) 이론은 만성적이고, 주기적인 스트레스는 개인의 자원을 손 실시키거나 완전히 소진시키는 원인이 되며[3] 과중한 업무 량이 소진에 영향을 미치는 가장 중요한 요인임을 밝혔다[4]. $\mathrm{COR}$ 이론을 근거로 볼 때 과중한 학업으로 지속적인 학업 스 트레스에 노출된 의과대학생들은 결국 자신이 가지고 있는 자원을 활용하지 못하고 최종적으로 학업 소진상태에 빠질 수 있음을 시사한다. 학업 소진은 장기간에 걸친 학업 스트레 스와 과도한 학업으로 인한 피로감, 좌절감, 학업에 대한 거리 감, 스트레스, 무력감, 학업에 대한 냉소적인 태도 등을 의미
Received: December 5, 2014 • Revised: Janurary 12, 2015 • Accepted: Janurary 14, 2015 Corresponding Author: Woo Taek Jeon (http://orcid.org/0000-0002-9213-6057)

Department of Medical Education, Yonsei University College of Medicine, 50-1 Yonsei-ro, Seodaemun-gu, Seoul 120-752, Korea

Tel: +82.2.2228.2510 Fax: +82.2.364.5450 email: wtjeon@yuhs.ac
Korean J Med Educ 2015 Mar; 27(1): 27-35. http://dx.doi.org/10.3946/kjme.2015.27.1.27 eISSN: 2005-7288

(C) The Korean Society of Medical Education. All rights reserved. This is an open-access article distributed under the terms of the Creative Commons Attribution Non-Commercial License (http:// creativecommons.org/licenses/by-nc/3.0/), which permits unrestricted non-commercial use, distribution, and reproduction in any medium, provided the original work is properly cited. 
한다[5].

국외 연구에 따르면 학업 소진은 의과대학생들의 $50 \% ~ 60 \%$ 가 경험하는 심리적 고통이며[6] 많은 학습량과 과도한 임상 실습이 소진과 관련 있다는 연구들이 꾸준히 보고되고 있다 [7,8]. 더불어 의학 분야에서의 성공을 예측하는 중요한 지표 [9]인 반면 학업 소진은 성별에 따라 차이가 있으며 특히 여학 생에게서 나타나는 소진현상은 자살률을 예측하는 중요한 지 표로 활용되고 있다[10]. 국내에서는 의과대학생의 학업 소진 연구가 시작단계로 최근에 학업 소진 척도 타당화 연구가 진 행된 바 있다[11]. 학업 소진은 세 가지 요인으로 구성되는데, 정서적 고갈은 지나친 학업에 대한 요구로 인한 심각한 피로 감을 의미하며, 냉소성은 학습에 대한 거리감을 의미한다. 무 능력감은 학업에서의 낮은 성취경험으로 인한 능력에 대한 회의감을 말한다[12]. 학업 소진 상태에 있는 학생들은 학업 에 대해 냉소적인 태도를 보이고 정서적으로 많이 지쳐있으 며 무능력감을 경험한다.

한편 학습자의 수행능력에 영향을 미치며 학업 성취를 가 장 잘 예언하는 개인차 변인은 학업적 자기효능감(academic self-efficacy)이다. 학업적 자기효능감은 학습자의 학업 수 행과 관련하여 학습자가 자신감을 가지고 과제 수행을 위해 필요한 행동을 조직하고 실행해 나가는 자신의 능력에 대한 판단을 의미한다. 학업적 자기효능감은 자신감, 자기조절효능 감, 어려운 과제 선호라는 세 가지 구인으로 구성되어있다. 자 신감이란, 자신의 학습능력에 대한 확신 또는 신념으로 정의 되며 자기조절효능감은 자기관찰, 자기판단, 자기반응과 같은 자기 조절적 기제를 잘 수행할 수 있는가에 대한 기대이다. 마 지막으로 어려운 과제 선호는 자신이 통제하고 다룰 수 있다 고 생각하는 도전적인 과제를 선택하는 것이다[13]. 사회인지 이론에 따르면 자기효능감은 어떤 일을 수행하는 데 있어 자 신의 능력뿐만 아니라 소진에 대한 지각에도 영향을 미친다. 즉, 자기효능감 수준의 변화는 소진과 같은 심리적 건강 (wellbeing) 상태의 변화와 매우 관련이 높다[14]. 소진은 효 능감 위기 또는 무능력감에서 발달하며 자신의 유능감 부족 에 대한 확신은 소진 발달에 결정적인 요인이 된다[15]. 자기 효능감과 소진과의 관련성을 조사한 연구들은 자기효능감이 부족한 사람들이 쉽게 소진되며 적응력이 부족하다는 것을 보고하고 있다[16]. 이 같은 결과는 자기효능감이 높을수록
학업 수행과 학업 성취는 커지며 소진이 낮아진다는 것을 시 사한다[4]. 이상의 선행 연구는 자기효능감과 소진과의 관련 성을 잘 설명해주고 있지만 학습자의 수행수준에 대한 예측 이 중요한 학습상황에서 개인의 성격특성으로서의 자기효능 감을 측정하는 것은 학생들의 학업지도나 상담에 유용한 정 보를 제공하는 데 한계가 있다[13]. 연구 결과 일반적인 자기 효능감과 학업성취도는 0.17 이라는 낮은 상관을 보였는데 이 는 일반적인 자기효능감보다는 학생들의 학업적 수행을 제대 로 예측할 수 있는 학업 수행과 관련된 맥락 특수적인 효능감 척도 사용이 필요함을 시사한다[17]. 이에 본 연구는 의과대 학생을 대상으로 학업 소진과 학업적 자기효능감과의 관계를 연구하고자 한다.

본 연구의 목적은 다음과 같다. 첫째, 남녀별 학업 소진의 차이를 밝히고자 한다. 국외 연구에서는 학업 소진에서 남녀 차이를 발견하지 못하거나 여학생들이 학업 소진을 더 많이 경험하는 것으로 보고하고 있다. 교육환경이 다른 국내에서 는 남녀별 학업 소진 현상이 어떤 양상을 나타내는지 밝혀보 고자 한다. 둘째, 외국과 달리 국내 의과대학에서는 의학과/ 의학전문대학원 시스템을 유지하면서 발달수준에 차이가 있 는 학생들을 동일한 교육과정으로 교육하고 있다. 본 연구는 의학과/의학전문대학원 학생들이 동일한 커리큘럼 안에서 학 업 소진과 학업 효능감을 경험하는 데 있어 차이가 있는지 분 석하고자 한다. 동시에 의학과/의학전문대학원 집단 간 학년 별 학업 소진의 차이도 함께 살펴보고자 한다. 마지막으로 낮 은 자기효능감이 소진을 유도한다는 이론적 배경에 근거해 학업적 자기효능감이 학업 소진에 미치는 영향을 분석하고자 한다.

\section{대상 및 방법}

\section{1. 연구 대상}

본 연구는 서울 소재 의과대학/의학전문대학원 병행 1 개 대학에 재학 중인 본과 $1,2,3,4$ 학년 총 475명을 대상으로 연구를 진행하였으며 회수가 안 되었거나 반응 질이 나쁜 설 문지를 제외하고 446 명의 자료를 분석에 사용하였다. 설문조 
Table 1. Subject Demographics ( $N=446)$

\begin{tabular}{clc}
\hline Variable & \multicolumn{1}{c}{ Classification } & No. of cases $(\%)$ \\
\hline Gender & Male & $296(66)$ \\
& Female & $150(34)$ \\
Group & Medical college student & $247(55)$ \\
& Graduate medical school student & $199(45)$ \\
Grade & 1st year & $129(29)$ \\
& 2nd year & $112(25)$ \\
& 3rd year & $109(24)$ \\
& 4th year & $96(22)$ \\
\hline
\end{tabular}

사 기간은 2013년 5월 2일부터 6월 27일까지 진행되었다. 설 문에 참여한 연구 대상자들의 인구학적 배경은 다음과 같다 (Table 1).

\section{2. 연구 방법}

\section{1) 학업 소진}

학업소진척도(Maslach Burnout Inventory-Student Survey, MBI-SS)를 국내 의과대학생 대상으로 타당화한 척도 [11]를 사용하였다. 학업소진척도는 총 14 문항이며 정서적 고 갈(5문항), 냉소성(4문항), 무능력감(역채점, 5 문항)으로 구성 되며 7점 척도로 평정한다(1, 전혀 아니다; 7 , 늘 그렇다). 정 서적 고갈과 냉소성, 무능력감의 점수가 높을수록 소진 상태 가 심각함을 의미한다. 본 연구에서의 전체 내적 일치도 (Cronbach $\alpha$ )는 0.86 이며 하위 요인별 내적 일치도는 정서 적 고갈 0.85 , 냉소성 0.83 , 무능력감 0.77 로 나타났다.

\section{2) 학업적 자기효능감}

학업적 자기효능감을 측정하기 위해서 Kim \& Park [13] 이 개발한 척도를 사용하였다. 총 26문항이며 6점 Likert척 도로 전혀 아니다(1점)에서 매우 그렇다(6점)까지 응답한다. 점수가 높을수록 학업적 자기효능감이 높음을 의미한다. 본 연구에서의 전체 내적 일치도(Cronbach $\alpha$ )는 0.88 , 자신감 0.84 , 어려운 과제 선호 0.91 , 자기조절효능감 0.77 로 나타났 다.

\section{3. 통계 분석}

각 변인들 간 관련성을 파악하기 위해 단순상관 분석을 실 시하였으며, 성별, 소속, 소속에 따른 학년별 학업 소진의 차 이를 분석하기 위해 다변량 분석을 시행하였다. 다음으로 학
업적 자기효능감이 학업 소진에 미치는 영향을 분석하기 위 해 회귀 분석을 실시하였다.

\section{결과}

\section{1. 변인 간 상관 분석}

학업적 자기효능감 하위 요인과 학업 소진 하위 요인 간 상 관 분석을 실시한 결과 모든 하위 요인에서 유의미한 부적 상 관(0.16-0.58)이 있는 것으로 나타났다(Table 2).

\section{2. 변인별 학업 소진의 차이}

\section{1) 성별, 소속별 학업 소진의 차이}

학업 소진이 성별(남녀), 소속(의학과, 의학전문대학원)별 차이가 있는지를 확인하기 위해 다변량 분석을 실시하였다. 분석 결과, 남녀별 학업 소진의 차이는 나타나지 않았다. 소속 별 학업 소진의 차이를 분석한 결과, 무능력감에서 의학과와 의학전문대학원 학생별 차이가 확인되었다(Table 3 ).

\section{2) 학년별 학업 소진의 차이}

학년별 학업 소진의 차이를 살펴보기 위해 의과대학생과 의학전문대학원생 집단으로 나누어 학년을 독립변인으로 하 고 학업 소진 하위 척도들을 종속변인으로 하는 다변량 분석 (multivariate analysis of variance)을 실시하였다. 분석 결 과, 의과대학생 집단은 학년에 따라 학업 소진 하위 척도에서 차이가 있는 것으로 나타났다(F $(9,1070)=3.28$, Wilks's lamda $=0.89, \mathrm{p}<0.001)$. 일변량 분석 결과, 정서적 고갈 (emotional exhaustion, EX)과 무능력감(inefficacy, IE)에 
Table 2. Correlations between Academic Burnout and Academic Self-Efficacy

\begin{tabular}{|c|c|c|c|c|c|c|c|c|}
\hline \multirow{2}{*}{ Factor } & \multicolumn{4}{|c|}{ Academic burnout } & \multicolumn{4}{|c|}{ Academic self-efficacy } \\
\hline & 1 & 2 & 3 & 4 & 5 & 6 & 7 & 8 \\
\hline EX & 1 & & & & & & & \\
\hline $\mathrm{CY}$ & $0.501^{* *}$ & 1 & & & & & & \\
\hline IE & $0.241^{* *}$ & $0.421^{* *}$ & 1 & & & & & \\
\hline$T$ of $B$ & $0.801^{* *}$ & $0.825^{* *}$ & $0.676^{* *}$ & 1 & & & & \\
\hline SC & $-0.456^{* *}$ & $-0.271^{* *}$ & $-0.351^{* *}$ & $-0.475^{* *}$ & 1 & & & \\
\hline SR & $-0.163^{* *}$ & $-0.332^{* *}$ & $-0.583^{* *}$ & $-0.441^{* *}$ & $0.293^{* *}$ & 1 & & \\
\hline TDP & $-0.336^{* *}$ & $-0.243^{* *}$ & $-0.391^{* *}$ & $-0.418^{* *}$ & $0.349 * *$ & $0.292^{* *}$ & 1 & \\
\hline$T$ of ASE & $-0.413^{* *}$ & $-0.376^{* *}$ & $-0.606^{* *}$ & $-0.593^{* *}$ & $0.737^{* *}$ & $0.709^{* *}$ & $0.744^{* *}$ & 1 \\
\hline Mean & 18.2 & 11.8 & 17.5 & 47.6 & 39.7 & 38.1 & 31.5 & 106.3 \\
\hline SD & 5.9 & 4.9 & 4.5 & 11.8 & 8.5 & 7.1 & 7.3 & 16.4 \\
\hline
\end{tabular}

EX: Emotional exhaustion, CY: Cynicism, IE: Inefficacy, T of B: Total score of burnout, SC: Self-confidence, SR: Self-regulatory, TDP: Task difficulty preference, $T$ of ASE: Total score of academic self-efficacy, SD: Standard deviation.

${ }^{* *} p<0.01$.

Table 3. Difference in Academic Burnout by Group and Gender

\begin{tabular}{|c|c|c|c|c|c|c|}
\hline \multirow{2}{*}{ Scale } & \multicolumn{3}{|c|}{ Gender difference } & \multicolumn{3}{|c|}{ Group difference } \\
\hline & Male $(n=296)$ & Female $(n=150)$ & $F(1.444)$ & MCS $(n=247)$ & GMSS (n=199) & $F(1.444)$ \\
\hline EX & $17.9 \pm 6.1$ & $18.9 \pm 5.4$ & 3.4 & $18.1 \pm 6.1$ & $18.4 \pm 5.6$ & 0.4 \\
\hline CY & $11.9 \pm 5.0$ & $11.5 \pm 4.5$ & 0.8 & $12.1 \pm 4.9$ & $11.4 \pm 4.8$ & 2.9 \\
\hline IE & $17.5 \pm 4.6$ & $17.5 \pm 4.3$ & 0.0 & $17.9 \pm 4.7$ & $16.9 \pm 4.1$ & $5.3^{*}$ \\
\hline
\end{tabular}

Data are presented as mean \pm standard deviation.

MCS: Medical college student, GMSS: Graduate medical school student, EX: Emotional exhaustion, CY: Cynicism, IE: Inefficacy. ${ }^{*} \mathrm{p}<0.05$.

Table 4. Multivariance Analysis of Academic Burnout Subscale by Year in Medical College Student

\begin{tabular}{lcccccc}
\hline \multirow{2}{*}{ Scale } & \multicolumn{5}{c}{ MCS } \\
\cline { 2 - 7 } & 1st year $(n=76)$ & 2nd year $(n=59)$ & 3rd year $(n=61)$ & 4th year $(n=51)$ & $F(3,243)$ & Bonferroni \\
\hline EX & $20.0 \pm 6.4$ & $18.2 \pm 5.4$ & $17.0 \pm 5.4$ & $16.0 \pm 6.3$ & $5.95^{* *}$ & 1 st $>3$ rd, 4 th \\
CY & $12.1 \pm 4.1$ & $13.2 \pm 5.1$ & $12.0 \pm 4.9$ & $11.1 \pm 5.4$ & 1.64 & \\
IE & $17.2 \pm 4.7$ & $19.0 \pm 4.0$ & $18.4 \pm 4.5$ & $16.9 \pm 5.2$ & $2.71^{*}$ & \\
\hline
\end{tabular}

Data are presented as mean \pm standard deviation.

MCS: Medical college student, EX: Emotional exhaustion, CY: Cynicism, IE: Inefficacy.

${ }^{*} p<0.05,{ }^{* *} p<0.01$.

서 학년별 차이가 나타났으며, Bonferroni 사후검증을 실시 한 결과, 정서적 소진에서 1학년과 3, 4학년 집단 간 유의한 차이가 있는 것으로 확인되었다(Table 4). 반면 의학전문대학 원생들은 학년에 따른 학업 소진의 차이가 유의하지 않은 것 으로 나타났다(Table 5).

\section{3. 학업적 자기효능감이 학업 소진에 미치는 영향}

학업적 자기효능감이 학업 소진에 미치는 영향을 알아보기 위해 회귀 분석을 실시하였다. 다중공선성 위배 여부를 확인 하기 위해 variance inflation factor (VIF), Tolerence값을 살펴본 결과 VIF는 1.14 1.19, Tolerence는 0.83 0.89로 나 
Table 5. Multivariance Analysis of Academic Burnout Subscale by Year in Graduate Medical School Student

\begin{tabular}{lccccc}
\hline \multirow{2}{*}{ Scale } & \multicolumn{5}{c}{ GMSS } \\
\cline { 2 - 6 } EX & 1st year $(n=53)$ & 2nd year $(n=53)$ & 3rd year $(n=48)$ & 4th year $(n=45)$ & $F(3,139)$ \\
CY & $19.7 \pm 5.7$ & $19.0 \pm 6.1$ & $17.6 \pm 4.7$ & $17.2 \pm 5.6$ & 2.20 \\
IE & $11.7 \pm 5.2$ & $11.3 \pm 5.0$ & $10.9 \pm 4.3$ & $11.5 \pm 4.4$ & 0.27 \\
\hline
\end{tabular}

Data are presented as mean \pm standard deviation.

GMSS: Graduate medical school student, EX: Emotional exhaustion, CY: Cynicism, IE: Inefficacy.

Table 6. Multiple Regression of Academic Burnout

\begin{tabular}{cccccccc}
\hline \multicolumn{2}{c}{ Independent variable } & $\mathrm{B}$ & $\mathrm{SE}$ & $\beta$ & $\mathrm{t}$ & $\mathrm{R}^{2}\left(\mathrm{Adj}_{-} \mathrm{R}^{2}\right)$ & $\mathrm{F}$ \\
\hline Academic self-efficacy & SC & -0.51 & 0.07 & -0.31 & $-7.58^{* * *}$ & & \\
& SR & -0.47 & 0.07 & -0.28 & $-6.99^{* * *}$ & $0.37(0.36)$ & $85.15^{* * *}$ \\
& TDP & -0.31 & 0.06 & -0.22 & $-5.40^{* * *}$ & & \\
\hline
\end{tabular}

SC: Self-confidence, SR: Self-regulatory, TDP: Task difficulty preference.

${ }^{* * *} p<0.001$.

타나 다중공선성이 존재하지 않음을 확인하였다. 또한 Durbin-Warson계수를 살펴본 결과 1.95 로 잔차가 독립적임 이 확인되었다. 회귀 분석을 실시한 결과 학업적 자기효능감 은 학업 소진에 대해 전체 $37 \%$ 의 설명력을 보였다 $(\mathrm{F}=85.15$, $\mathrm{p}<0.000)$. 구체적으로 살펴보면 자신감 $(\beta=-0.31, \mathrm{p}<0.000)$, 자기조절효능감 $(\beta=-0.28, p<0.000)$, 어려운 과제 선호 $(\beta=$ $-0.22, \mathrm{p}<0.000)$ 는 학업 소진을 유의미하게 예언하였다 (Table 6).

\section{고찰}

본 연구는 국내에서 아직까지 활발히 연구가 진행되고 있 지 않은 의과대학생의 학업 소진 현상을 분석하고자 하였다. 이를 위해 남녀별/소속별/학년별 학업 소진의 차이를 살펴보 고 학업적 자기효능감이 학업 소진에 미치는 영향을 분석하 고자 하였다. 그 결과는 다음과 같다.

첫째, 학업적 자기효능감과 학업 소진 하위 요인 간 상관 분 석을 실시한 결과, 학업적 자기효능감과 학업 소진은 부적상 관 $(\mathrm{r}=-0.59)$ 이 있는 것으로 나타났으며 상관계수가 0.6 수준 으로 두 변인 간 상당한 관련성이 있음이 확인되었다. 본 연구 결과는 학업 소진과 학업적 자기효능감과의 관계를 보고한
선행 연구[18]와 일치한다. 그리고 학업 소진과 자기효능감과 의 상관을 -0.3 수준으로 보고한 연구[19]와 비교할 때 자기 효능감보다 학업적 자기효능감이 학습자의 학업 수행을 예측 하는 데 있어 효과적인 변인임을 확인할 수 있었다. 상관 분석 결과는 학생들의 학업 소진 예방을 위해 학업에서 자신감을 잃지 않도록 지도해야 함을 시사한다.

둘째, 성별 학업 소진의 차이를 분석한 결과, 남녀별 통계적 으로 유의미한 차이는 나타나지 않았다. 이 같은 결과는 학업 에서 경험하는 소진은 남녀 구분 없이 의과대학생이면 누구 나 경험할 수 있음을 시사한다. 그러나 통계적 의미는 없지만 평균값을 비교해보면 정서적 고갈에서 여학생들의 평균이 남 학생들에 비해 높은 것을 확인할 수 있다. 국외 연구에서도 의 과대학 재학중인 여학생이 남학생보다 정서적 고갈을 더 많 이 경험한다는 연구 결과가 보고된 바 있다[10]. 또한 여성 의 료인(의과대학생, 의사)에게서 발견되는 정서적 고갈과 냉소 성의 초기 징후들은 자살률을 예측하는 지표임이 확인된 바 있다[8]. 정서적 고갈과 냉소성을 경험하는 여성의료인들이 모두 자살을 선택하는 것은 아니겠지만 여성 의료인이 정서 적 소진에 취약하다는 사실을 간과해서도 안 될 것이다. 추후 연구에서는 성별 차이에 대한 지속적인 연구가 진행될 필요 가 있으며 특히 여학생들이 겪는 정서적 고갈의 실체를 밝히 는 작업이 필요하겠다. 또한 현재 정서적 고갈을 경험하고 있 
Su Hyun Lee and Woo Taek Jeon: The relationship between academic self-efficacy and academic burnout in medical students

는 의과대학생들을 위한 다양한 상담 및 치료 프로그램 개발 및 시스템 정비가 필요하다.

셋째, 소속별 학업 소진의 차이를 분석한 결과, 의과대학생 들이 의학전문대학원생들보다 학업에서 무능력감을 더 경험 하는 것으로 나타났다. 의과대학생들이 의학전문대학원생들 보다 학업에서 무능력감을 더 경험한다는 것은 의과대학 교 육과정에 시사하는 바가 크다. 연구에 따르면 의학전문대학 원생들이 인지적 학습 전략과 시간관리 전략을 더 많이 사용 하며 특히 심화학습과 관련된 인지 전략을 더 많이 사용한다 [20]. 의학전문대학원 학생들은 이미 4년간의 학부과정 동안 우수한 학업 성취를 경험한 학생들이다. 이들은 4년간의 학부 과정에서 습득한 지식과 의과대학에서 새롭게 습득한 지식을 정교하게 연결하고 비판적 사고를 통해 새로 습득한 지식을 검증하고 증례를 통해 분석해 내는 심화 전략을 사용하기 때 문에 학업에서 무능력감을 적게 경험하는 것으로 유추할 수 있다. 단순암기 중심의 학습 전략은 증례 중심의 학습에 적합 한 전략이 아니기 때문에 만족할 만한 성과를 내기 어렵다. 본 연구에서 나타난 의과대학생과 의학전문대학원생들 간의 차 이는 단순히 4년이라는 시간의 차이나 개인이 가지고 있는 능 력의 차이로 보기 어렵다. 4년이라는 기간 동안 학생들이 어 떤 교육적 경험을 했는가에서 그 해답을 찾아야 할 것이다. 의 과대학생들이 학업에서의 무능력감을 극복하기 위해서는 예 과 2년 교육과정에 대한 발상의 전환이 필요하다. 본과 1학년 들의 학업 스트레스가 가장 심하다는 선행 연구 결과[21]는 예과 교육과정이 본과 1학년 학습을 준비하기 위한 발판 역할 을 하지 못하고 있음을 시사한다. 대학마다 의예과 교육에 기 대하는 목표가 다르기 때문에 다양한 내용으로 교육과정을 운영하지만 본과 교육과 단절된 교육과정은 학생들이 본격적 으로 의학을 공부하는 본과에 진입했을 때 학업 장벽을 만들 고 학업에서의 무능력감을 조장할 수 있다는 것을 인지할 필 요가 있다.

넷째, 의과대학생과 의학전문대학원생들은 발달적 차이가 있는 집단이기 때문에 의과대학생과 의학전문대학원생 집단 을 나누어 학년별 학업 소진의 차이를 분석하였다. 그 결과 의 과대학생 집단에서는 정서적 고갈과 무능력감에서 학년 간 유의한 차이가 나타났으며 특히 정서적 고갈은 3,4 학년보다 1 학년들이 더 많이 경험하는 것으로 나타났다. 반면 의학전문
대학원생들은 학년에 따른 학업 소진의 차이가 유의하지 않 은 것으로 나타났다. 정서적 고갈은 소진을 구성하는 가장 중 요한 요인으로 과도한 학습량, 환경에 대한 통제와 자율성 상 실로 생겨난다[22]. 실제로 학업에서의 과부하는 정서적 고갈 을 정의하는 중요한 기준으로 학생들이 하루의 많은 시간을 학업에 사용하면서 극도의 피로감과 정서적 고갈을 경험한다 는 것을 확인한 바 있다[23]. 그리고 시험이 의과대학생들의 소진에 영향을 미치는 가장 큰 스트레스 요인임을 밝혔다 [24]. 본 연구를 위해 설문조사를 진행했던 5 6월 기간 동안 본과 1 학년은 성적에 반영되는 지필시험을 5 회 치렀으며, 본 과 2학년 2번, 본과 3, 4학년은 0 회였으며 학기 초부터 누적 된 시험 횟수를 살펴보면 1학년 10회, 2학년 2회, 4학년 2회, 3 학년 0회로 확인되었다. 학년별 시험 횟수를 비교해 볼 때, 본과 1 학년들이 잦은 시험에 노출되면서 정서적 고갈을 더 많 이 경험하고 있는 것으로 유추할 수 있다. 통계적으로 유의하 지는 않았지만 의학전문대학원생들도 1 학년들의 정서적 고갈 평균 점수가 가장 높았다. 즉, 1 학년들은 의과대학생과 의학 전문대학원생들 간 정도의 차이는 있지만 모두 심리적/신체 적 피로감을 경험하고 지쳐있는 상태인 것이다. 본과 1학년 과정은 주로 기초의학을 배우는 시기인데 1학년에 집중된 많 은 시험 횟수와 버거운 학습량은 학생들이 학업은 물론 기초 의학에 대한 관심과 흥미를 가지는 데 부정적인 영향을 미칠 수 있다. 본과 1학년들의 정서적 고갈을 해결하기 위해서는 기초학 과목의 일부를 예과 2학년 교육과정과 연계하거나 1 , 2학년 강의를 기초/임상 통합 과목으로 운영하여 기초와 임 상지식을 균형적으로 쌍을 수 있도록 조정할 필요가 있겠다. 본 연구 결과는 학업 소진이 교육과정 운영과 상당히 밀접한 관련이 있음을 의미하며 학교마다 학년에 따른 학업 소진이 다양한 양상으로 나타날 수 있음을 시사한다. 예를 들어 Galán et al. [25]의 연구에서 3학년과 6학년(우리나라 본1과 본4)의 소진을 비교한 결과 정서적 고갈과 냉소성은 6학년(본 4)들이 더 많이 경험하고 무능력감은 3학년(본1)들이 더 많이 경험하는 것으로 나타났다. 국내외 연구에서 학년 간 학업 소 진의 차이는 통계적으로 유의한 결과를 보이지만 학년별 경 험하는 소진 내용은 다르게 나타나고 있기 때문에 그 차이를 규명하기 위해서는 추가적인 연구가 계속 진행될 필요가 있 겠다. 한편 의과대학생들의 경우 무능력감에서 학년별 차이 
가 유의한 것으로 나타났다. 사후 분석은 통계적으로 유의미 한 차이가 없는 것으로 나타나 학년별 비교는 무의미하지만 단순 평균 비교를 하자면 2학년>3학년>1학년>4학년 순으 로 무능력감을 경험하는 것으로 나타났다. 의과대학생들이 경험하는 무능력감의 내용과 원인을 밝히기 위해서는 추가적 인 연구가 필요하다.

다섯째, 학업적 자기효능감이 학업 소진을 $37 \%$ 설명하는 것으로 나타났다. 학업 소진을 설명하는 개인차 변인 중 단일 변인으로 $37 \%$ 의 설명력을 갖는다는 것은 학업적 자기효능감 이 학업 소진을 잘 설명해주는 변인임을 의미한다. 특히 학업 적 자기효능감 하위 요인 중 자신감 $(\beta=-0.31)$ 이 학업 소진 에 가장 크게 영향을 미치는 예언변인으로 나타났다. 즉, 학업 에서의 자신감이 낮을수록 학업 소진에 빠질 가능성이 높아 지는 것이다. 선행 연구 결과 학교부적응 행동(낮은 학업 동 기와 학업 성취, 잦은 결석, 학업 중단)의 주된 원인이 학업 소진이며[4] 자신감 상실 및 열등감이 유급의 심리적 요인 [26]으로 확인된 바 있다. 선행 연구 결과를 종합하면, 학업에 서의 낮은 자신감과 열등감이 학업 소진과 학업 부적응을 예 측할 수 있는 변인임을 시사한다. 우수한 학업 성적으로 의과 대학에 입학한 학생들이 학업에 대한 자신감을 잃는다는 것 은 교육과정 운영과 평가 측면에서 시사하는 바가 있다. 평가 는 학습 동기를 높이기 위한 목적으로 활용되어야 하며 상대 평가를 하던, 절대평가를 하던지 간에 중요한 것은 학생이 배 우고 있고 알아가고 있으며 학문적으로 성장하고 있다는 것 을 경험하게 하는 평가가 이루어져야 한다는 것이다. 학생들 이 평가를 통해 자신이 아는 것과 모르는 것을 확인하고 앞으 로 어떻게 공부를 해야 하는지 방향을 잡을 수 있다면 학업에 서 자신감을 상실하지는 않을 것이며 궁극적으로 학업 소진 을 예방할 수 있을 것이다. 따라서 대학은 교수개발을 통해 교 수들의 교수(teaching) 및 평가역량을 개발하는 데 주력해야 할 것이며 나아가 교육전담 트랙을 만들어 교육 및 평가를 전 문적으로 담당할 수 있는 인적자원을 지속적으로 개발할 필 요가 있겠다. 학생들이 자기주도적 학습을 하기 위해서는 시 간관리능력, 정서조절능력, 현실적인 계획능력 및 실행능력 등이 선행되어야 한다. 따라서 대학에서는 시간관리, 공부계 획, 자기인식 및 정서조절과 관련된 다양한 상담 및 교육 프로 그램을 제공할 수 있는 학생지원시스템을 갖출 필요가 있다.
본 연구는 학업 소진 연구에서 불모지라고 할 수 있는 의과 대학생을 대상으로 학업적 자기효능감이 학업 소진을 설명하 는 예언변인임을 확인하였다는 점에서 의의를 찾을 수 있겠 다. 그러나 본 연구는 몇 가지 제한점을 가지고 있는데 미래 연구에 대한 제언과 함께 논의하면 다음과 같다. 첫째, 의과대 학마다 교육과정 운영체계에서 다소 차이가 있기 때문에 일 개 의과대학의 사례로 의과대학생들의 일반적인 학업 소진 현상을 설명하는 데 한계가 있다. 추후 많은 대학에서 소진 연 구가 진행되어 국내 의과대학생들의 일반적인 학업 소진 양 상을 연구할 필요가 있겠다. 둘째, 의과대학생들의 학업 소진 은 시험 횟수나 임상실습의 강도와 같은 교육과정과 매우 밀 접한 관련이 있기 때문에 학업 소진을 측정하는 시점이 결과 에 영향을 미칠 수 있다. 추후 다양한 시점에서 학업 소진 연 구들이 진행될 필요가 있겠다.

\section{Acknowledgements: None.}

Funding: None.

Conflicts of interest: None.

\section{REFERENCES}

1. Masten R, Tusak M, Zalar B, Ziherl S. Stress, coping and social support in three groups of university students. Psychiatr Danub 2009; 21: 41-48.

2. Tosevski DL, Milovancevic MP, Gajic SD. Personality and psychopathology of university students. Curr Opin Psychiatry 2010; 23: 48-52.

3. Freedy JR, Hobfoll SE. Stress inoculation for reduction of burnout: a conservation of resources approach. Anxiety Stress Coping 1994; 6: 311-325.

4. Yang HJ. Factors affecting student burnout and academic achievement in multiple enrollment programs in Taiwan's technical-vocational colleges. Int J Educ Dev 2004; 24: 283-301.

5. Gao HY. The differences in academic burnout and 
Su Hyun Lee and Woo Taek Jeon: The relationship between academic self-efficacy and academic burnout in medical students

academic failure tolerance between motivation types. Asian J Educ 2012; 13: 125-147.

6. Dyrbye LN, Thomas MR, Eacker A, Harper W, Massie FS Jr, Power DV, Huschka M, Novotny PJ, Sloan JA, Shanafelt TD. Race, ethnicity, and medical student well-being in the United States. Arch Intern Med 2007; 167: 2103-2109.

7. Guthrie E, Black D, Bagalkote H, Shaw C, Campbell M, Creed F. Psychological stress and burnout in medical students: a five-year prospective longitudinal study. J R Soc Med 1998; 91: 237-243.

8. Dyrbye LN, Thomas MR, Massie FS, Power DV, Eacker A, Harper W, Durning S, Moutier C, Szydlo DW, Novotny PJ, Sloan JA, Shanafelt TD. Burnout and suicidal ideation among U.S. medical students. Ann Intern Med 2008; 149: 334-341.

9. Tartas M, Walkiewicz M, Majkowicz M, Budzinski W. Psychological factors determining success in a medical career: a 10-year longitudinal study. Med Teach 2011; 33: el63-el72.

10. Fang DZ, Young CB, Golshan S, Moutier C, Zisook S. Burnout in premedical undergraduate students. Acad Psychiatry 2012; 36: 11-16.

11. Lee SH, Lee DY. Validation of the MBI-SS Scales-based on medical school students. Asian J Educ 2013; 14: 165-187.

12. Pagnin D, De Queiroz V, De Oliveira Filho MA, Gonzalez NV, Salgado AE, Cordeiro e Oliveira B, Lodi CS, Melo RM. Burnout and career choice motivation in medical students. Med Teach 2013; 35: 388-394.

13. Kim AY, Park IY. Construction and validation of academic self-efficacy scale. Korean J Educ Res 2001; 39: 95-123.

14. Salanova M, Agut S, Peiró JM. Linking organizational resources and work engagement to employee performance and customer loyalty: the mediation of service climate. J Appl Psychol 2005; 90: 1217-1227.
15. Cherniss C. Role of professional self-efficacy in the etiology and amelioration of burnout. In: Schaufeli W, Maslach C, Marek T, eds. Professional burnout: recent developments in theory and research. Washington, DC, USA: Taylor \& Francis; 1993. p 135-149.

16. Hobfoll SE, Freedy J. Conservation of resources: a general stress theory applied to burnout. In: Schaufeli W, Maslach C, Marek T, eds. Professional burnout: recent developments in theory and research. Washington, DC, USA: Taylor \& Francis; 1993. p 115-129.

17. Kim AY. A study on the academic failure-tolerance and its correlates. Korean J Educ Psychol 1997; 11: 1-19.

18. Jo HJ, Lee JY, Jang JY. The mediating effects of academic self-efficacy and academic failure tolerance on the relationship between excessive academic demands and academic burnout on highschool students. Korean J Couns 2013; 14: 1605-1621.

19. Charkhabi M, Azizi Abarghuei M, Hayati D. The association of academic burnout with self-efficacy and quality of learning experience among Iranian students. Springerplus 2013; 2: 677-681.

20. Shin HI, Jeon WT, Yang EB. Relationship between learning strategies and academic achievement in medical college and graduate medical school students. Korean J Med Educ 2010; 22: 197-204.

21. Lee KH, Ko Y, Kang KH, Lee HK, Kang J, Hur Y. Mental health and coping strategies among medical students. Korean J Med Educ 2012; 24: 55-63.

22. Santen SA, Holt DB, Kemp JD, Hemphill RR. Burnout in medical students: examining the prevalence and associated factors. South Med J 2010; 103: 758-763.

23. Montero-Marin J, Monticelli F, Casas M, Roman A, Tomas I, Gili M, Garcia-Campayo J. Burnout syndrome among dental students: a short version of the "Burnout Clinical Subtype Questionnaire" adapted for students (BCSQ-12-SS). BMC Med Educ 2011; 11: 103-113.

24. Backović DV, Zivojinović JI, Maksimović J, Maksimović 
M. Gender differences in academic stress and burnout among medical students in final years of education. Psychiatr Danub 2012; 24: 175-181.

25. Galán F, Sanmartín A, Polo J, Giner L. Burnout risk in medical students in Spain using the Maslach Burnout
Inventory-Student Survey. Int Arch Occup Environ Health 2011; 84: 453-459.

26. Kim SH, Jeon WT. The failure experiences of medical school students: a qualitative study. Korean J Med Educ 2008; 20: 351-362. 